OPEN ACCESS

Edited by:

Mario Tavazza,

Energia Nucleare ed Energie

Alternative (ENEA), Italy

Reviewed by:

Carl Deom

University of Georgia, Georgia

Emanuela Noris,

Consiglio Nazionale delle Ricerche

(CNR), Italy

${ }^{*}$ Correspondence:

Peng Zhang

zhangpeng@sibs.ac.cn

Specialty section:

This article was submitted to

Plant Microbe Interactions,

a section of the journa

Frontiers in Plant Science

Received: 06 June 2017 Accepted: 14 September 2017 Published: 27 September 2017

Citation:

Bi H, Fan W and Zhang P (2017) C4

Protein of Sweet Potato Leaf Curl

Virus Regulates Brassinosteroid Signaling Pathway through Interaction with AtBIN2 and Affects Male Fertility in Arabidopsis.

Front. Plant Sci. 8:1689 doi: 10.3389/fpls.2017.01689

\section{C4 Protein of Sweet Potato Leaf Curl Virus Regulates Brassinosteroid Signaling Pathway through Interaction with AtBIN2 and Affects Male Fertility in Arabidopsis}

\author{
Huiping Bi ${ }^{1,2}$, Weijuan Fan ${ }^{1}$ and Peng Zhang ${ }^{1 *}$ \\ 1 National Key Laboratory of Plant Molecular Genetics, CAS Center for Excellence in Molecular Plant Sciences, Institute of \\ Plant Physiology and Ecology, Shanghai Institutes for Biological Sciences, Chinese Academy of Sciences, Shanghai, China, \\ ${ }^{2}$ Key Laboratory of Systems Microbial Biotechnology, Tianjin Institute of Industrial Biotechnology, Chinese Academy of \\ Sciences, Tianjin, China
}

Sweepoviruses have been identified globally and cause substantial yield losses and cultivar decline in sweet potato. This study aimed to investigate the interaction between sweepovirus and plant host by analyzing the function of the viral protein C4 of Sweet potato leaf curl virus-Jiangsu (SPLCV-JS), a sweepovirus cloned from diseased sweet potato plants in East China. Ectopic expression of the C4 in Arabidopsis altered plant development drastically with phenotypic changes including leaf curling, seedling twisting, deformation of floral tissues and reduction of pollen fertility, and seed number. Using bimolecular fluorescence complementation analysis, this study demonstrated that the SPLCV-JS C4 protein interacted with brassinosteroid-insensitive 2 (AtBIN2) in the plasma membrane of Nicotiana benthamiana cells. The C4 AtBIN2 interaction was further confirmed by yeast two-hybrid assays. This interaction led to the re-localization of AtBIN2-interacting proteins AtBES1/AtBZR1 into the nucleus which altered the expression of brassinosteroid (BR)-response genes, resulting in the activation of BRsignaling pathway. The interaction of SPLCV-JS C4 and AtBIN2 also led to the downregulated expression of key genes involved in anther and pollen development, including SPROROCYTELESS/NOZZLE, DEFECTIVE IN TAPEL DEVELOPMENT AND FUNCTION 1, and ABORTED MICROSPORES, which caused abnormal tapetal development, followed by defective exine pattern formation of microspores and pollen release. Consequently, male fertility in the $C 4$ transgenic Arabidopsis was reduced. The present study illustrated how the sweepovirus C4 protein functioned in host cells and affected male fertility by interacting with the key components of BR-signaling pathway.

Keywords: Arabidopsis, AtBIN2, BR-signaling pathway, C4 protein, interaction, male fertility, sweet potato leaf curl virus 


\section{INTRODUCTION}

Sweepoviruses, named for the sweet potato begomoviruses, are monopartite (no DNA-B, alpha-satellite, or beta-satellite components associated) and phylogenetically distinct from the Old and New World begomovirus groups (Clark et al., 2012). Recently, increasing strains of whitefly transmitted sweepoviruses, mainly sweet potato leaf curl viruses, have been identified globally (Trenado et al., 2011; Wasswa et al., 2011; Albuquerque et al., 2012; Bi and Zhang, 2012; Clark et al., 2012; Pardina et al., 2012; Liu et al., 2014). Besides sweet potato (Ipomoea batatas), sweepoviruses also infect other Ipomoea species worldwide, such as I. nil or I. setosa (Trenado et al., 2011), and have been reported to cause substantial yield losses and cultivar decline regionally in some sweet potato varieties (Clark and Hoy, 2006; Ling et al., 2010; Gibson and Kreuze, 2015). As a vegetatively propagated root crop, virus infections in sweet potato often build up over generations. Besides, sweet potato infected by sweepovirus in combination with other virus species is not uncommon (Cuellar et al., 2014). Thus, the build-up and combined infection of viruses provide opportunities for pseudo-recombination and interaction events, a process beneficial to virus evolution and diversity.

The host-pathogen interaction of geminivirus in plants has been extensively studied over the past 20 years. Many reports have shown that geminiviral proteins participate and affect various biological processes of plant cells, including modifying cell cycle regulation, cell proliferation, interaction with the host cell defense system, plant development by interacting with host development-related proteins and cell-to-cell migration of virus through plasmodesmata (reviewed by Lozano-Durán et al., 2011; Hanley-Bowdoin et al., 2013; Lucioli et al., 2016). Like other monopartite geminiviruses, sweepoviruses have small, circular, single-stranded DNA genomes. The DNA virion-sense strand encodes two proteins (V1/coat protein CP and V2), and the complementary strand encodes four proteins. The replicationassociated protein ( $\mathrm{C} 1$ or Rep) and the replication-enhancer protein (C3 or REn) are required for viral DNA replication, and the transcriptional activator protein (C2 or TrAP) has been implicated in the control of viral gene expression. The C4 protein, the smallest protein whose coding sequence is entirely contained within the Rep coding region in a different open-reading frame, plays multiple functions in virus-plant interactions (Hanley-Bowdoin et al., 2013). The C4 of Beet severe curly top virus (BSCTV) can up-regulate the RING finger protein to affect BSCTV infection by regulating the host cell cycle (Lai et al., 2009). In some monopartite geminiviruses, C4 has been shown to be associated with the development of disease symptoms, such as leaf curling and vein swelling (Stanley and Latham, 1992; Krake et al., 1998; Gutierrez, 2002). $\mathrm{C} 4$ also functions as a suppressor of geminivirus-induced gene silencing in plants (Vanitharani et al., 2004; Gopal et al., 2007; Dogra et al., 2009). The C4 proteins of Beet curly top virus (BCTV) were shown to interact with 7 of the 10 members of the Arabidopsis thaliana SHAGGY-like protein kinase (AtSK) family (AtSK11, AtSK12, AtSK13, AtSK21, AtSK22, AtSK23, and AtSK32) (Piroux et al., 2007; Deom and Mills-Lujan, 2015; Mills-Lujan et al., 2015) and inhibit phosphorylation of BRI1EMS-suppressor 1 (BES1). The interactions between $\mathrm{C} 4$ protein and SHAGGY-like protein kinases were also confirmed for monopartite Tomato leaf curl virus (ToLCV) (Dogra et al., 2009) and bipartite Tomato golden mosaic virus (TGMV) (Piroux et al., 2007).

A. thaliana SHAGGY-like protein kinases are homologs of the evolutionarily conserved glycogen synthase kinase 3 (GSK3) family of serine/threonine kinases in animals (Doble and Woodgett, 2003). The AtSK gene family has evolved into 10 members possessing diverse functions, of which 7 members have been implicated in the brassinosteroid (BR) signaling pathway. Some of these AtSKs were reported to have redundant roles in BR signaling. Among all the AtSKs, AtSK21 (also known as AtBIN2) acts as a key negative regulator of BR signaling (Vert and Chory, 2006; Ryu et al., 2007; Youn and Kim, 2015). As a class of essential plant hormones, BRs are implicated in regulating broad aspects of plant growth and development, including vegetative and reproductive development, germination, senescence, and responses to various biotic and abiotic stresses (Yang et al., 2011; Fàbregas and Caño-Delgado, 2014; Youn and Kim, 2015). Recent models suggest that AtBIN2 targets two closely related transcription factors, AtBES1 and AtBZR1, which regulate the expression of BR target genes (reviewed by Wang et al., 2012). In the absence of BRs, AtBIN2 acts as an active kinase to phosphorylate and inactivate AtBES1/AtBZR1, which is retained in the cytosol by 14-3-3 proteins or degraded by $26 \mathrm{~S}$ proteasome (Vert and Chory, 2006; Gampala et al., 2007). In the presence of $\mathrm{BR}$, the hormone binds to $\mathrm{BR}$ receptor kinase (BRI1), which initiates a signaling cascade that results in the activation of BRI1suppressor 1 (BSU1), a phosphatase that inactivates AtBIN2 by dephosphorylating a conserved phosphotyrosine residue. The inhibition of AtBIN2 allows transcription factors BES1/BZR1 to be dephosphorylated by protein phosphatase 2A (PP2A), which accumulate in the nucleus and recruit proteins such as BIM1 and Myb30 to form diverse transcriptional complexes, which bind to promoter regions of BR-responsive genes and consequently regulate their expression (Yang et al., 2011).

Recently, a sweepovirus, Sweet potato leaf curl virus-Jiangsu (SPLCV-JS), was identified from diseased sweet potato in East China. Infectivity analysis showed that SPLCV-JS could cause mild symptoms in both Nicotiana benthamiana and sweet potato, and this virus could synergize with heterologous betasatellite DNA to enhance symptom severity and viral DNA accumulation (Bi and Zhang, 2012, 2014). A few studies to date have focused on molecular mechanisms of sweepoviruses, with the exception of epidemic warning (Simmons et al., 2009), genome identification (Albuquerque et al., 2012), and infectivity assays (Trenado et al., 2011; Bi and Zhang, 2014). This study focused on the function analysis of the C4 protein of SPLCVJS to further investigate the interaction between sweepovirus and plant hosts. Since geminivirus C4 protein could interact with several members of AtSKs in plants and the phenotype of the SPLCV C4-expressing Arabidopsis lines was similar to the mutants of BR-signaling pathway genes (Li et al., 2001), this study hypothesized that SPLCV C4 might regulate BR-signaling 
pathway by interacting with BR-related proteins. This study showed that $\mathrm{C} 4$ interacted with AtBIN2 in vivo, resulting in the activation of BR-signaling pathway with phenotypic changes, including the abnormal development of tapetum and pollen grains, leading to reduced male fertility in the C4-expressing Arabidopsis plants. It demonstrated how SPLCV-JS C4 protein interacted with plants by activating the BR-signaling pathway.

\section{MATERIALS AND METHODS}

\section{SPLCV-JS C4 Protein Sequence Analysis}

The amino acid sequence of SPLCV-JS (GenBank accession No. FJ176701) C4 was aligned with selected geminivirus C4 protein sequences from GenBank using the ClustalX program. After multiple sequence alignments, phylogenetic analysis was performed with MEGA software version 4.0 using the neighborjoining method and the bootstrap option (1000 replicates) (Tamura et al., 2007).

\section{PLASMID CONSTRUCTION}

All primers used for gene cloning and plasmid construction are listed in Supplementary Table S1. The SPLCV-JS C4 gene was amplified by polymerase chain reaction (PCR) from the SPLCV-JS genome using the primer pair C4-5FPK/C4-3RPP. The PCR product was digested with KpnI and Pst I, and ligated into the binary vector pCAMBIA1301 (Cambia, Canberra, Australia), which was digested with the same enzymes to construct pCAMBIA1301-C4. Similarly, the full-length sequence of AtBIN2 amplified from the cDNA of Arabidopsis seedlings with the primer pair AtBIN2-5FPK/AtBIN2-3RPP was cloned into the vector pCAMBIA1301 using KpnI and PstI sites to construct pCAMBIA1301-AtBIN2.

For yeast two-hybrid assays, the $C 4$ was amplified with the primer pair C4-5FPNde/C4-3RPP2, and cloned into the vector pGBKT7 (Clontech) to form a fusion protein with the GAL4 DNA-binding domain (pGBKT7-C4). The N-terminal C4N55 was amplified with the primer pair C4-5FPNde/C4N55$3 \mathrm{RP}$ and cloned into the vector pGBKT7 via double digestion with NdeI and PstI to form pGBKT7-C4N55. The C-terminal C4C31 was amplified with primers C4C31-5FPNde and C43RPP2 and cloned into pGBKT7 via NdeI and PstI sites to generate plasmid pGBKT7-C4C31. AtBIN2 was amplified from the cDNA of Arabidopsis seedlings with the primer pair AtBIN2-5FPNde/AtBIN2-3RPE and then ligated into pGADT7 (Clontech) through $\mathrm{NdeI}$ and EcoRI sites to form a fusion protein with the GAL4 activation domain (pGADT7-AtBIN2).

For the bimolecular fluorescence complementation (BiFC) assay, the C4 was PCR-amplified and digested with BamHI and SpeI and ligated into vector 35S-N1-YFPN to generate an in-frame C-terminal fusion to the YFP N-terminal fragment downstream of the CaMV 35 S promoter (C4-YFPN). AtBIN2 was PCR-amplified and digested with $B a m \mathrm{HI}$ and EcoRI and ligated into vector 35S-N1-YFPC to generate an in-frame C-terminal fusion to the YFP C-terminal fragment (AtBIN2-YFPC).
For subcellular localization, AtBES1 and AtBZR1 were PCRamplified without the stop codon, digested with KpnI and NotI and cloned into an intermediate cloning vector that was tagged with eGFP at the C-terminal. Then, the AtBES1-eGFP and the AtBZR1-eGFP fragments were amplified by AtBES15FPK/eGFP-3RPB or AtBZR1-5FPK/eGFP-3RPB, respectively, and digested with $K p n \mathrm{I}$ and BglII to ligate into the KpnI- and BamHI-digested expression vector pCAMBIA1301.

\section{Generation of Transgenic Arabidopsis Lines}

Arabidopsis thaliana ecotype Columbia (Col0) was transformed following standard protocols (Zhang et al., 2006). T1 seeds were collected and germinated on selection media and then plantlets were transplanted to soil in a growth chamber $\left(22 \pm 1^{\circ} \mathrm{C}, 16 \mathrm{~h}\right.$ light/8 h dark photoperiod). The transgene copy number was confirmed using the Southern blot analysis (Sambrook et al., 2001).

\section{DNA Extraction and Southern Blot Analysis}

The total genomic DNA was extracted from the transgenic Arabidopsis plants according to the method developed by Soni and Murray (1994). For the Southern blot analysis, an aliquot of $10 \mu \mathrm{g}$ of the genomic DNA from each sample was digested with HindIII and analyzed using a standard protocol for Southern blot (Sambrook et al., 2001). Digested genomic DNA was hybridized with DIG-labeled probe specific to the SPLCV-JS C4 gene. Labeling, hybridization, and chemiluminescent detection were performed according to the instructions of the manufacturer (Roche Applied Science, Germany).

\section{Yeast Two-Hybrid Assay}

For SPLCV-JS C4 self-activation assay, yeast strain AH109 (Clontech) was transformed with pGBKT7-C4, pGBKT7-C4C31, pGBKT7-C4N55, and the pGBKT7 control. For the C4 and AtBIN2 interaction assay, plasmids pGBKT7-C4, pGBKT7C4C31, and pGBKT7-C4N55 were co-transformed with plasmid pGADT7-AtBIN2 into the yeast strain AH109. A yeast colony co-transformed with pGBKT7-53 and pGADT7-T plasmids was used as a positive control in the experiment. A yeast strain co-transformed with pGBKT7-lam and pGADT7-T was used as a negative control. All transformants were grown on a dropout medium lacking tryptophan, leucine, histidine, and adenine

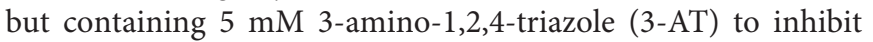
residual HIS3 expression. The expression of $L a c Z$ reporter gene was initially monitored using $\mathrm{X}-\alpha-\mathrm{Gal}$ as the $\beta$-galactosidase substrate.

\section{BiFC Assay for SPLCV-JS C4 and AtBIN2 Interaction}

Nicotiana benthamiana plants were grown in pots at $23^{\circ} \mathrm{C}$ with a $16 \mathrm{~h}$ light $/ 8 \mathrm{~h}$ dark photoperiod and 60\% humidity. Before agroinfiltration, the solutions of Agrobacterium tumefaciens harboring binary C4-YFPN or AtBIN2-YFPC were mixed equally. $N$. benthamiana plants grown to the four to five 
leaf stages were agroinfiltrated with the bacterial suspension using a syringe. $N$. benthamiana plants agroinfiltrated with A. tumefaciens harboring C4-YFPN and YFPC or YFPN and AtBIN-YFPC were used as negative controls. The agroinfiltrated leaves were observed under a laser confocal microscope (Carl Zeiss, Jena, Germany) at $36 \mathrm{~h}$ postinfiltration (hpi).

\section{Subcellular Localization of AtBES1/AtBZR1}

$N$. benthamiana leaves were agroinfiltrated with the A. tumefaciens harboring the expression vectors AtBES1eGFP and AtBZR1-eGFP, separately. The suspensions of A. tumefaciens harboring the expression vector AtBES1eGFP/AtBZR1-eGFP and pCAMBIA1301-AtBIN2 were mixed equally and used for agroinfiltration to illustrate the effects of AtBIN2 and C4 on the subcellular localization of AtBES1/AtBZR1. In addition, the suspensions of A. tumefaciens harboring the expression vector AtBES1-eGFP/AtBZR1-eGFP, pCAMBIA1301-AtBIN2, and pCAMBIA1301-C4 were mixed equally for the agroinfiltration experiment. All agroinfiltrated leaves were observed under a laser confocal microscope (36 hpi).

\section{BR Sensitivity Analysis}

Arabidopsis seeds were surface-sterilized and sown on Murashige and Skoog (MS) agar plates with $30 \mathrm{~g} / \mathrm{L}$ sucrose. The plates were cold-treated for 2 days at $4^{\circ} \mathrm{C}$. For root growth inhibition assays, MS plates were placed in a vertical orientation for 4 days in a growth chamber at $23^{\circ} \mathrm{C}$ with a $16 \mathrm{~h}$ light $/ 8 \mathrm{~h}$ dark photoperiod and $60 \%$ humidity, and the seedlings were then transferred to MS plates amended with $1 \mu \mathrm{M}$ epibrassinolide (BL) for 5 days. The root length was measured before and after BL treatment, and the hormone sensitivity was measured as root growth inhibition.

\section{Quantitative Real-Time Reverse Transcription-PCR Analysis}

The total RNA was extracted from Arabidopsis seedlings or floral buds containing approximately stages 7-9 anthers using the Tiangen Plant RNAprep Pure Kit (Tiangen, Beijing, China). The first-strand cDNA was synthesized using the Takara PrimeScript First-Strand cDNA Synthesis Kit (TaKaRa, Dalian, China) and then used for real-time quantitative reverse transcriptionPCR (qRT-PCR) analysis. Primer sequences for each gene are listed in Supplementary Table S2. PCR reactions were performed in a $20 \mu \mathrm{L}$ volume containing $2 \times$ SYBR Green Master Mix (TOYOBO Co., Ltd.), 50 ng cDNA, 400 nM forward primer, and $400 \mathrm{nM}$ reverse primer in a Bio-Rad CFX96 thermocycler. Actin or a U-BOX gene At5g15400 (Ye et al., 2010) was used as a reference control for seedlings or anthers, respectively. All samples were assayed in triplicate and repeated at least twice. The relative expression levels were calculated using the $2^{-\Delta \Delta \mathrm{Ct}}$ method (Livak and Schmittgen, 2001).

\section{Pollen Viability Assay Using Fluorescein Diacetate Staining and Pollen Germination Rate Analysis}

Anthers at the anthesis stage were removed and used to brush pollens into the fluorescein diacetate (FDA) staining buffer on the slide. After staining for $20 \mathrm{~min}$, the slide was observed under an optical microscope in blue light (wavelength $=495 \mathrm{~nm}$ ), and viable pollen grains emitted fluorescence. FDA staining was performed as described by Li (2011). Nikon NIS-Elements BR 3.0 software was used to calculate the pollen viability.

Arabidopsis flowers that were just open were picked and the pollen grains were dusted on the solid pollen germination medium ( $\mathrm{Li}$ et al., 1999) and allowed to germinate for 3-5 h or overnight at room temperature. The pollen germination was observed under a microscope Nikon TE2000-S, and the germination ratio was calculated using Nikon NIS-Elements BR 3.0 software.

\section{Semi-Thin Section and Light Microscopy Observation}

Arabidopsis inflorescences were incubated for at least $12 \mathrm{~h}$ in formaldehyde-acetic acid-ethanol (FAA) fixing solution (10\% formaldehyde, 5\% acetic acid, and $47.5 \%$ ethanol in water), dehydrated in a graded ethanol series $(2 \times 50,60,70,85$, and $95 \%$, and $3 \times 100 \%$ ), embedded in epoxy resin and sectioned. Anther transverse sections were stained with $1 \%$ toluidine blue at $42^{\circ} \mathrm{C}$ for 1-2 h (Sanders et al., 1999), bright-field photographs were taken using a Nikon TE2000-S microscope.

\section{Scanning and Transmission Electron Microscopy}

For scanning electron microscopic (SEM) analysis, wild-type and mutant inflorescences were fixed overnight in FAA, dehydrated in a graded ethanol series as described earlier, and samples were critical-point dried in liquid $\mathrm{CO}_{2}$. Individual anthers and pollen from flowers that corresponded to a specific stage of wild-type anther development were mounted on SEM stubs. Mounted samples were coated with palladium-gold and then examined under an Autoscan SEM JMS-6360 LV (JEOL, Tokyo, Japan).

For transmission electron microscopic (TEM) analysis, the buds approximately containing anthers at stage 9 were collected and fixed in $2.5 \%$ glutaraldehyde in phosphate buffer $(\mathrm{pH} 7.2)$ for $4 \mathrm{~h}$ at $4^{\circ} \mathrm{C}$. After fixation, the tissue was washed and postfixed with $1 \% \mathrm{OsO}_{4}$ overnight at $4^{\circ} \mathrm{C}$. The samples were then dehydrated in a graded ethanol series, infiltrated with a graded series of epoxy resin in epoxy propane, and embedded in Epon 812 resin. Ultrathin sections were stained with $1 \%$ uranyl acetate, followed by lead citrate solution and viewed under a TEM (HITACHI H-7650; Hitachi, Tokyo, Japan).

\section{Statistical Analysis}

All data were presented as the mean \pm standard deviation (SD) of at least three independent experiments. Statistical analysis was performed using the SPSS statistical package, version 15.0 (SPSS, IL, United States). Student's $t$-test was used for analyzing the significance of the differences between the wild-type and 
transgenic Arabidopsis lines. A value of $P \leq 0.05$ or $P \leq 0.01$ was indicated by an asterisk $\left({ }^{*}\right)$ or double asterisks $\left({ }^{* *}\right)$, respectively.

\section{RESULTS}

\section{Overexpression of SPLCV-JS C4 Protein Alters Arabidopsis Development}

The 258-bp long open-reading frame of SPLCV-JS C4 encoded a $9.2-\mathrm{kDa}$ protein consisting of 85 amino acids. The amino acids BLAST analysis showed that $\mathrm{C} 4 / \mathrm{AC} 4$ proteins were diverse among different strains/isolates. The most similar ones were chosen to perform phylogenetic analysis. Most branches of the phylogenetic tree were less than 70 bootstrap values and the SPLCV-JS C4 protein was grouped together with other sweepoviruses (e.g., SPLCV, SPLCJV, and SPLCBV) (Supplementary Figure S1).

Further sequence alignment of SPLCV-JS C4 was performed with three well-studied $\mathrm{C} 4$ proteins (also known as AC4 in bipartite begomoviruses), including C4 of BCTV, AC4 of TGMV, and AC4 of East African cassava mosaic Cameroon virus (EACMCV). The N-terminal $\mathrm{G}^{2}$ site in a conserved consensus $\mathrm{N}$-myristoylation motif was required for membrane binding of both the bipartite begomoviruses EACMCV AC4 (Fondong et al., 2007) and the monopartite geminivirus BCTV C4 (Piroux et al., 2007). Besides, the four residues $\mathrm{P}^{44}, \mathrm{~A}^{45}, \mathrm{~S}^{49}$, and $\mathrm{P}^{50}$ within the central domain of BCTV were reported to contribute to pathogenicity (Piroux et al., 2007). Mills-Lujan et al. (2015) showed that $S^{49}$ was phosphorylated, and this phosphorylation was required for BCTV C4 function. SPLCV C4 showed several conserved amino acid residues, including $\mathrm{G}^{2}, \mathrm{P}^{44}, \mathrm{~S}^{49}$, and $\mathrm{P}^{50}$ (Figure 1).

Transgenic A. thaliana lines expressing the C4 protein were produced to better characterize the developmental defects induced by the $\mathrm{C} 4$ in planta in a system more amenable to genetic studies. The $C 4$ gene was expressed under the control of the CaMV $35 S$ promoter in order to have constitutive expression. After transformation, none of the transgenic Arabidopsis T1 generation showed a segregation ratio of 3:1, indicating that the transgene integration was not a single insertion. Indeed, Southern blot analysis showed that all the transgenic lines were multi-copy insertion (Supplementary Figure S2A). Three independent transgenic lines, C4-11, C4-36, and C4-37, were chosen for further study. Real-time qRT-PCR analyses showed that the expression level of $\mathrm{C} 4$ in C4-11 and C4-37 was three or two times higher than that of C4-36 (Supplementary Figure S2B). Compared with the wild type (Figure 2A), C4transgenic seedlings showed elongated and twisted petioles and severely curled leaves (Figures 2B-D). During growth, the whole plantlet was distorted and deformed, as illustrated by the C4-11 plant line (Figure 2E). Different from Col0 in flowering status (Figures 2E-I), the flower buds of the C4 transgenic plants were not completely enclosed by the sepals and the early flowers were poorly organized (Figures 2J,K). The anthers were shrunk (compare Figure $\mathbf{2 H}$ with Figure $\mathbf{2 L}$ ), and the siliques of the transgenic lines were distorted and had fewer seeds compared with the wild type after destaining (Figure 2M). The phenotype severity was in accordance with the C4 expression level in C411, C4-36, and C4-37 seedlings (Supplementary Figure S2B), in which C4-11 had the highest $\mathrm{C} 4$ expression level with the most severe phenotype.

SPLCV-JS C4 protein was divided into two segments, the N-terminal C4N55 and C-terminal C4C31, to study the functional domains of $\mathrm{C} 4$. Both truncated genes were expressed under the control of CaMV $35 S$ promoter in Arabidopsis. The phenotype of the homozygote Arabidopsis is shown in Supplementary Figure S3. No obvious difference was observed between the C4N55 transgenic Arabidopsis and the wild-type control. However, the differences between the C4C31 transgenic Arabidopsis and the wild-type control were mild, including slightly curved leaves and plants, distorted flowers, and slightly distorted siliques (Supplementary Figure S3). These results demonstrated that the occurrence of severe developmental defects in the transgenic Arabidopsis was correlated with the expression of the N-terminal domain of SPLCV-JS C4. Attempts were also made to generate transgenic sweet potato expressing the C4 protein, but no transgenic plants could be produced using the routine protocol (Yang et al., 2011), suggesting that the expression of the SPLCV-JS C4 might be lethal.

\section{SPLCV-JS C4 Protein Interacts with AtBIN2 to Retain AtBES1/AtBZR1 in the Nucleus and Consequently Up-regulated the BR-Signaling Pathway in Arabidopsis}

The interaction between SPLCV-JS C4 protein and AtSK21 (AtBIN2) was confirmed by the yeast two-hybrid assay (Figure 3A). The yeast strain co-transformed with the $C 4$ and AtBIN2 could grow well in the dropout medium (SD/-Trp/-His/Ade) supplemented with $15 \mathrm{mM} 3-\mathrm{AT}$ and X- $\alpha$-Gal, and was blue, in both combinations pGBKT7-C4 + pGADT7-AtBIN2 and pGADT7-C4 + pGBKT7-AtBIN2 (Figure 3A). When C4 was truncated into two domains, $\mathrm{N}$-terminal C4N55 and C-terminal C4C31, the C4C31 could interact with AtBIN2 weakly, and the C4N55 did not interact with AtBIN2 (Figure 3A). Using the $\mathrm{BiFC}$ assay, it was further investigated whether the SPLCVJS C4 protein interacted with the AtBIN2 in plant cells. The C4 alone was localized in both the plasma membrane and the nucleus of N. benthamiana protoplasts and epidermal cells as described in a previous study (Bi and Zhang, 2012). AtBIN2 was previously reported both in the cytoplasm and on the plasma membrane (Vert and Chory, 2006). After agroinfiltration of the constructs expressing C4 fused to YFP (C4-YFPN) and AtBIN2 fused with YFP (AtBIN2-YFPC) into intact $N$. benthamiana leaves, a strong fluorescent signal could be detected mainly in the plasma membrane of the co-infiltrated leaves 36 hpi (Figure 3B, left panel). No detectable signal was noted in the negative controls (Figure 3B, middle and right panels). The result indicated that SPLCV-JS C4 protein interacted with AtBIN2 in vivo, and the interaction mainly occurred at the periphery of the cell.

AtBIN2 phosphorylated transcription factors BES1/BZR1 to target them for degradation in the proteasome (Yang et al., 2011). However, AtBIN2 activity is inhibited in the presence of BR. Subsequently, unphosphorylated BES1/BZR1 accumulated in the 


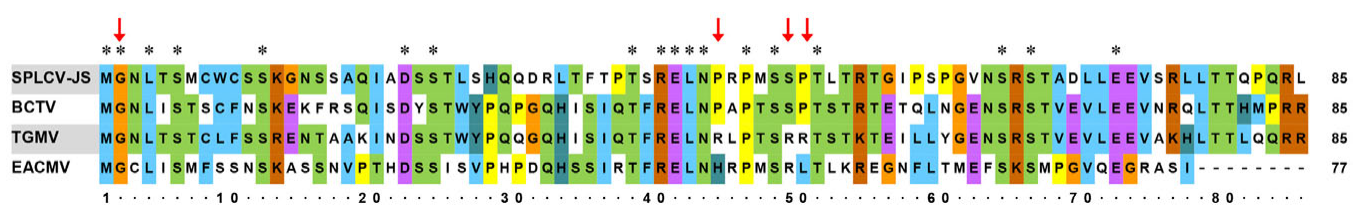

FIGURE 1 | Comparison of amino acid sequences of SPLCV-JS C4 proteins with those of other geminiviruses. Conserved amino acid residues among these sequences are indicated by the asterisks and key amino acid residues related to geminivirus C4 function by red arrows. BCTV, Beet curly top virus; TGMV, Tomato golden mosaic virus; and EACMCV, East African cassava mosaic Cameroon virus.
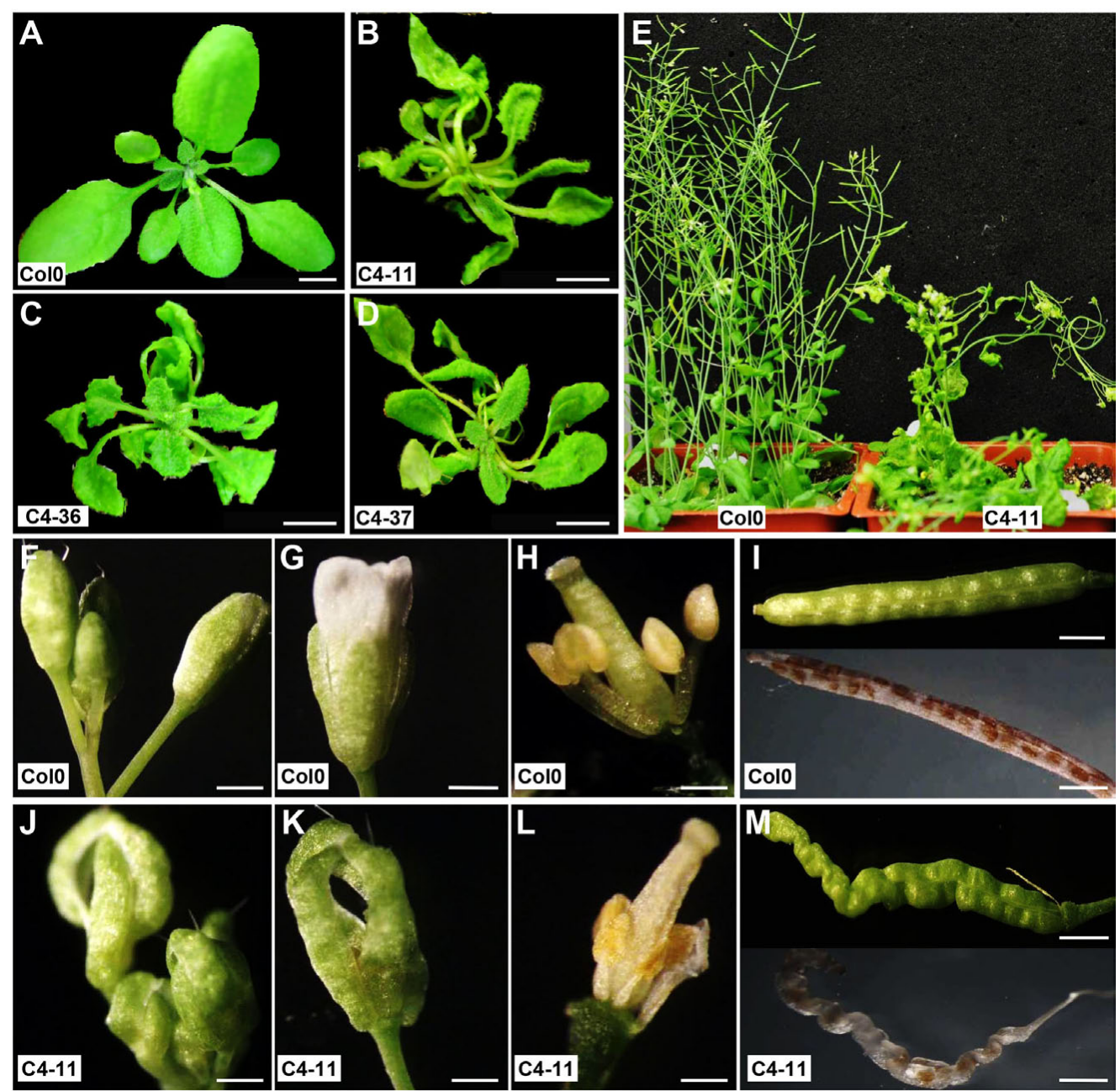

FIGURE 2 | Phenotypic changes of SPLCV-JS C4 transgenic Arabidopsis plant lines. (A-D) Seedlings of wild-type Col0 and transgenic lines C4-11, C4-36, and C4-37. (E) Flowering Arabidopsis plants of wild-type ColO and C4-11. (F-M) Inflorescence, flowers, stamens, pistils, and siliques of wild-type Col0 (F-I) and C4-11 (J-M). Scale bar = $1 \mathrm{~cm}(\mathbf{A}-\mathbf{D})$ and $1 \mathrm{~mm}$ (F-M).

nucleus and recruited proteins such as BIM1 and Myb30 to form diverse transcriptional complexes to regulate the expression of BR-response genes (Yang et al., 2011). The pattern of AtBES1 and AtBZR1 in the presence or absence of the C4 and/or AtBIN2 was studied to better understand the working mode between SPLCV $\mathrm{C} 4$ and AtBIN2 interacting proteins.

Both AtBES1 and AtBZR1 tagged with eGFP protein were localized in the nucleus in the $N$. benthamiana epidermal cells (Figures 4A,B), different from the eGFP control where fluorescence was reported in the cytoplasm and nucleus (Figure 4C). When co-infiltrated with $\mathrm{C} 4$, the patterns of AtBES1-eGFP and AtBZR1-eGFP in the $N$. benthamiana epidermal cells were unchanged 36 hpi (Figures 4D,E) in comparison with the eGFP control (Figure $\mathbf{4 F}$ ), indicating that C4 protein did not directly affect the subcellular localization of AtBES1/AtBZR1. When AtBES1-eGFP or AtBZR1-eGFP were 


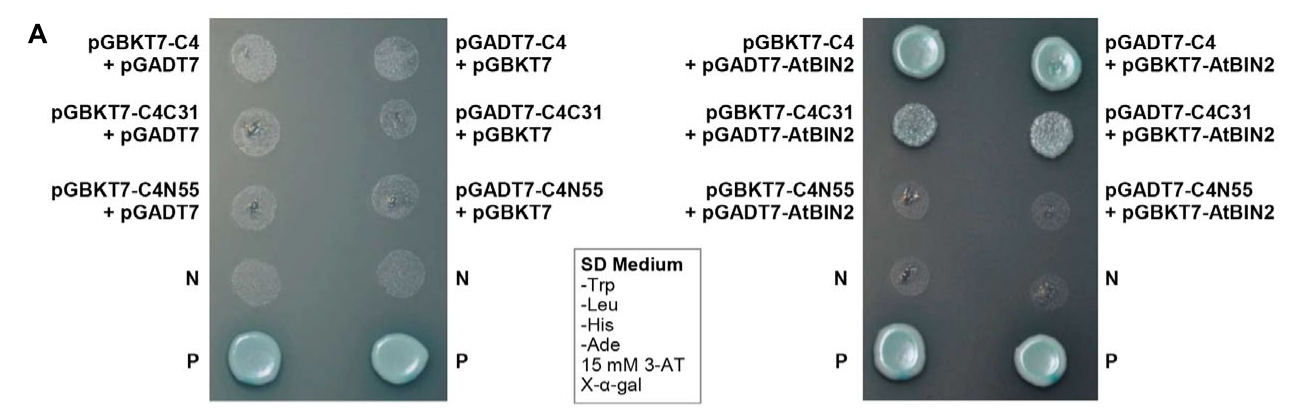

B

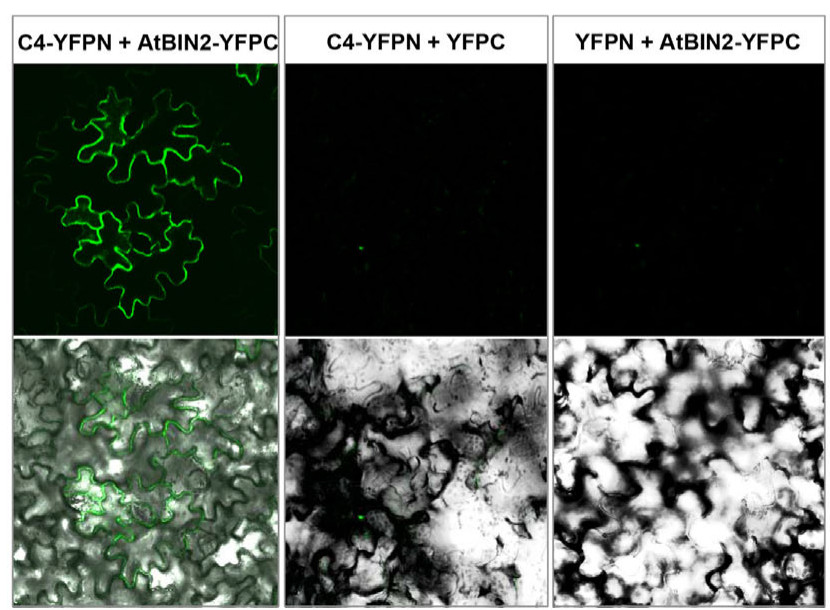

FIGURE 3 | SPLCV-JS C4 and AtBIN2 interaction. (A) The interaction of SPLCV-JS C4 with AtBIN2 detected using the yeast two-hybrid assay. Yeast clones grown on dropout medium with $\mathrm{X}$ - $\alpha$-galactosidase $(X-\alpha$-Gal) and 3-aminotriazole (3-AT, $15 \mathrm{mM})$ but lacking tryptophan, leucine, histidine, and adenine

(SD/-Trp/-leu/-His/-Ade). P, Positive control (pGBKT7-53 and pGADT7-T co-transformant); N, pGBKT7 empty vector. (B) Bimolecular fluorescence complementation analysis of the interaction between SPLCV-JS C4 and AtBIN2 in agroinfiltrated Nicotiana benthamiana leaf epidermal cells. Leaves were co-infiltrated with C4-YFPN and AtBIN2-YFPC. Leaves co-infiltrated with C4-YFPN and YFPC or YFPN and AtBIN2-YFPC were used as controls. Images were acquired by laser confocal microscopy at $36 \mathrm{hpi}$.

co-infiltrated with AtBIN2, both AtBES1-eGFP and AtBZR1eGFP were mainly localized at the cell periphery and in the cytoplasm (Figures $\mathbf{4 G}, \mathbf{H}$ ), suggesting that the proteins were phosphorylated and relocalized from the nucleus to the cytoplasm. This finding was in accordance with previous reports that AtBIN2 could phosphorylate AtBES1/AtBZR1 and target them for degradation in the proteasome (Gampala et al., 2007; Ryu et al., 2007). In contrast, when AtBES1-eGFP or AtBZR1eGFP were co-infiltrated with AtBIN2 and SPLCV-JS C4, the fluorescent signal was detected only in the nucleus (Figures $4 \mathbf{I}, \mathbf{J}$ ), suggesting that the $\mathrm{C} 4$ protein reversed the effects of AtBIN2 on the subcellular localization of AtBES1/AtBZR1 and maintained the accumulation of these two transcription factors in the nucleus.

The effects of $\mathrm{C} 4$ on the phenotype and the expression of two BR biosynthetic genes CPD and DWF4, which were regulated by AtBES1/AtBZR1 (He et al., 2005; Yang et al., 2011), were further studied in the presence of BL. After treatment with $1 \mu \mathrm{M} \mathrm{BL}$, the wild-type Arabidopsis showed severely curled leaves, elongated and twisted petioles, and shorter taproot with more secondary roots (Figure 5C), similar to the C4expressing lines (Figures 2B-D). Real-time qRT-PCR indicated that before $\mathrm{BL}$ treatment, the expression of $C P D$ and DWF4 was much lower in the C4-expressing lines than the wild type. After BL treatment, the gene expression of both $C P D$ and DWF4 was repressed to a lower level in all the C4-expressing lines and the wild type, compared with mock-treated samples (Figures 5A,B). These data showed that the phenotypes with curled leaves, elongated and twisted petioles, and repressed expression of CPD and DWF4 of C4-expressing lines were similar to those of the wild-type plants treated with $\mathrm{BL}$. These results indicated that, just like the case of BL-treated plants, the BRsignaling pathway was activated in the C4-expressing Arabidopsis lines.

\section{SPLCV-JS C4 Protein Affects Microsporogenesis and Male Fertility in Arabidopsis}

The microsporogenesis and pollen morphology of C4 transgenic lines were studied to further characterize the impact of SPLCVJS C4 on Arabidopsis development. With FDA staining, the pollen viability of C4-11, C4-36, and C4-37 was 15.9, 24.5, and $22.0 \%$, respectively, which was significantly lower than that 


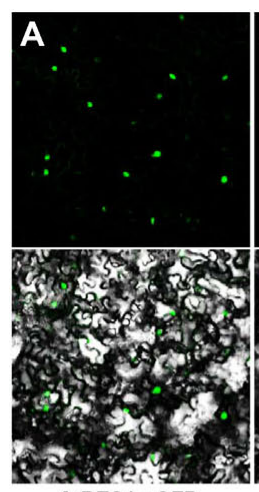

AtBES1-eGFP

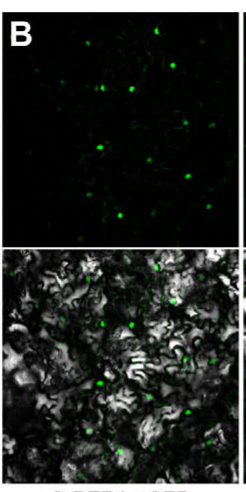

AtBZR1-eGFP

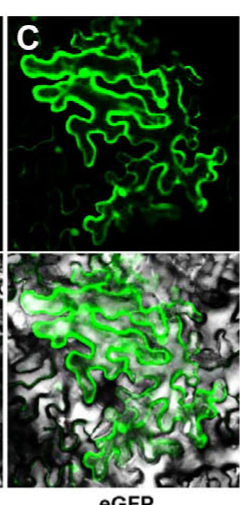

eGFP

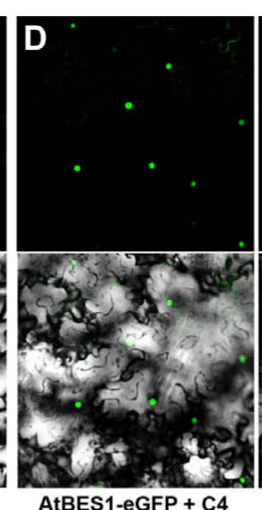

AtBES1-eGFP + C4

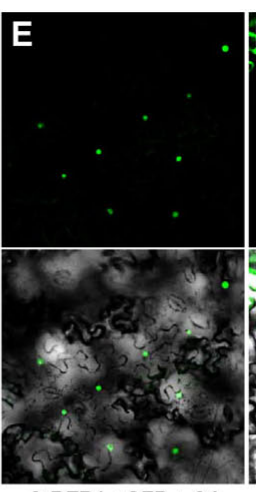

AtBZR1-eGFP + C4

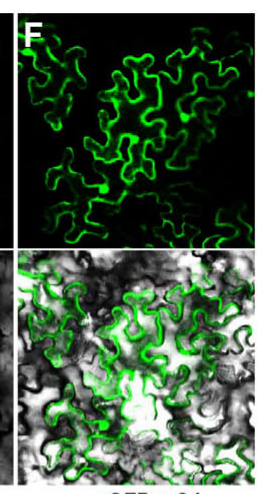

eGFP + C4

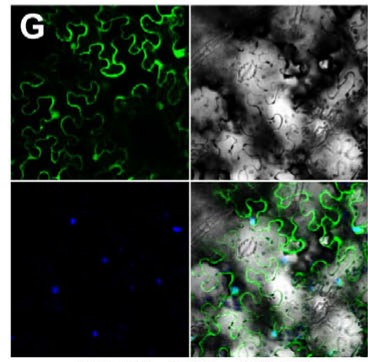

AtBES1-eGFP + AtBIN2

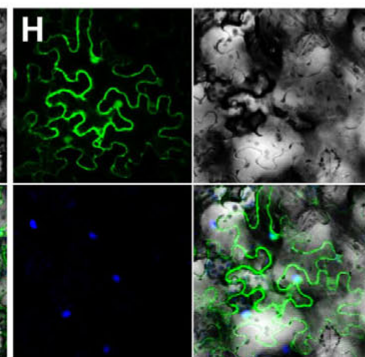

AtBZR1-eGFP + AtBIN2

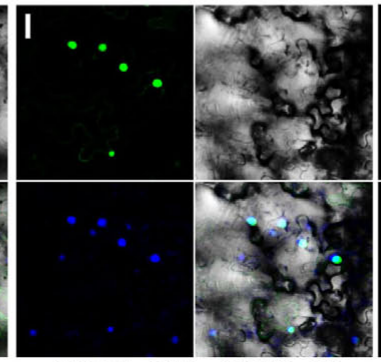

AtBES1-eGFP + AtBIN2 + C4

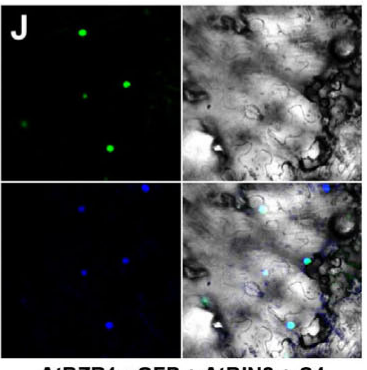

AtBZR1-eGFP + AtBIN2 + C4

FIGURE 4 | Subcellular localizations of AtBES1/AtBZR1 in N. benthamiana epidermal cells in the presence of AtBIN2 and SPLCV-JS C4 proteins. (A,B) The nuclear localization of AtBES1 and AtBZR1 using eGFP-fused proteins. (C) The eGFP control. (D-F) Co-infiltration of AtBES1-eGFP, AtBZR1-eGFP, or eGFP with SPLCV-JS C4. $\mathbf{( G , H ) ~ T h e ~ c h a n g e d ~ l o c a l i z a t i o n ~ o f ~ A t B E S 1 ~ a n d ~ A t B Z R 1 ~ w h e n ~ c o - i n f i l t r a t e d ~ w i t h ~ A t B E S 1 - e G F P ~ o r ~ A t B Z R 1 - e G F P ~ w i t h ~ A t B I N 2 . ~ ( I , J ) ~ T h e ~ r e - l o c a l i z a t i o n ~ o f ~ A t B E S 1 ~}$ and AtBZR1 to the nucleus when co-infiltrated with AtBES1-eGFP or AtBZR1-eGFP together with AtBIN2 and SPLCV-JS C4. Images were acquired by laser confocal microscopy 36 hpi. GFP fluorescence, bright-field channel, and DAPI stained merged images are shown, accordingly.

of wild type (64.9\%, Supplementary Figure S4). The pollen germination ratio of C4-11, C4-36, and C4-37 was 26.4, 30.8, and $26.7 \%$, respectively, which was also significantly lower than the $64.4 \%$ of wild type (Supplementary Figure S4). The anthers of the C4-expressing Arabidopsis lines usually contained fewer pollen grains than that of the wild type. Transverse section of anthers at stage 9 showed that the microspore numbers of C4-11, C4-36, and C4-37 were reduced compared with that of the wild type. Meanwhile, the shape of many microspores of the transgenic lines was abnormal and vacuolated (Figure 6).

The anthers at the anthesis stage were also observed by SEM analysis. At this stage, the dehiscent anthers of the wild type contained few pollen grains (Figure 7A). A considerable number of pollen grains remained in the inner wall of $\mathrm{C} 4$ 11, C4-36, and C4-37 anthers (Figures 7B-D), indicating that these $\mathrm{C} 4$-expressing lines were deficient in pollen release after anther dehiscence, similar to the previously reported BR-related mutants that were defective in pollen release (Ye et al., 2010). Further observation showed that the inner surface of dehisced anthers from C4-11, C4-36, and C4-37 was similar to that of the wild type, but the exines of the pollen grains of the transgenic lines were abnormal (Figures 7E-H). Under higher magnification, the wild-type exine had a network-like structure with many lacunae and three narrow apertures (Figure 7I). In contrast, the lacunae of C4-11, C4-36, and C4-37 lacked the regularity exhibited by the wild type, and some of the apertures of the transgenic lines were sunken (Figures 7J-L). Further examination of pollen development using TEM analysis showed that the microspores of C4-11, C4-36, and C4-37 at stage 9 were poorly organized compared with that of the wild type (Figure 7M), and the exine was irregular (Figures $7 \mathbf{N}-\mathbf{P}$ ). The complete bacula/tectum structure in the wild type was deficient in the transgenic lines (Figures 7Q-T). Moreover, the bacula/tectum structure had almost disappeared in some transgenic plants (Figures $7 \mathbf{R}-\mathbf{T}$ ). The abnormal structure of the exine in the $C 4$ transgenic lines could explain the deficiency of pollen grains release from the inner surface of the anther wall, much like the bri1-116 mutant of Arabidopsis (Ye et al., 2010).

To investigate the morphological changes that lead to pollen grain defects in the transgenic lines, the anther and microspore morphology of the C4-11, C4-36, and C4-37 lines was examined using TEM. At stage 5, the cell arrangements of tapetum and microspore mother cells of C4-11, C4-36, and C4-37 were irregular compared with those of the wild type (Figures 8A-D), and their tapetal cells were larger and more vacuolated also (Figures $8 \mathrm{E}-\mathbf{H}$ ). At stage 8 , a number of vesicles containing fibrous materials could be found in the tapetum of the wild type (Figure 8I), while the tapetum of the transgenic lines was still significantly vacuolated (Figures $\mathbf{8 J}-\mathbf{L}$ ). Since tapetum is responsible for the synthesis and secretion of exine material, 

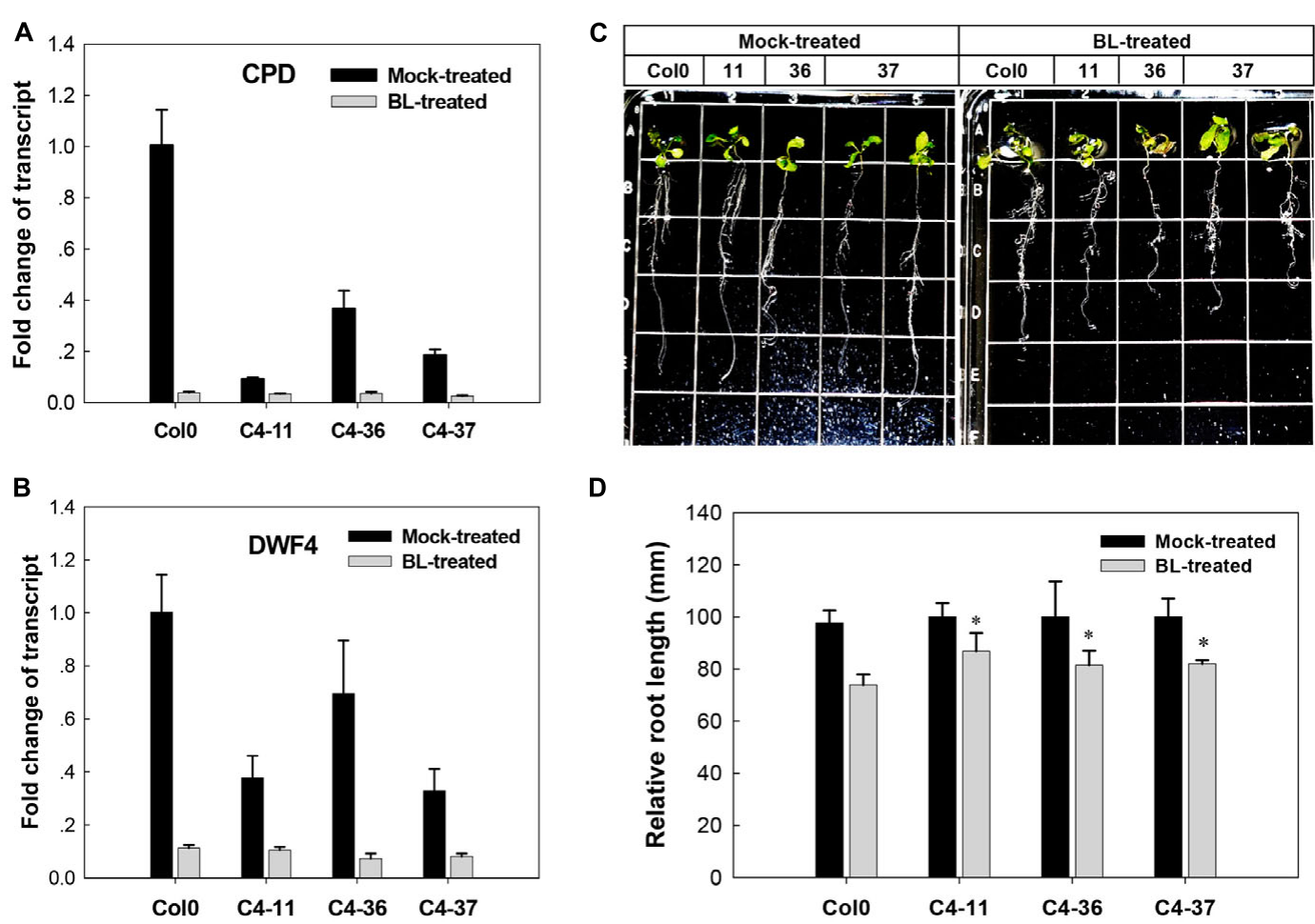

D

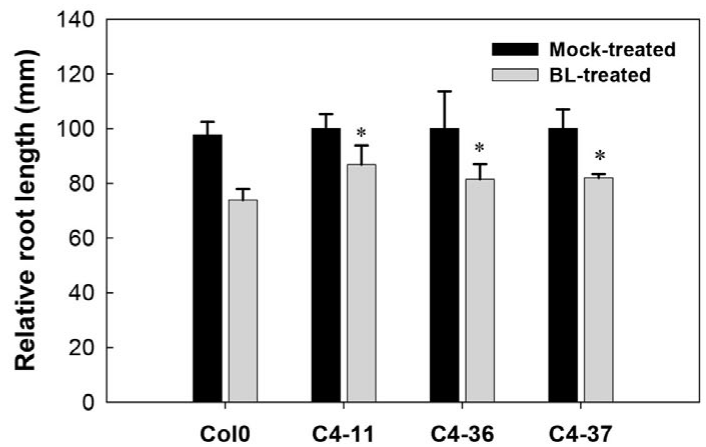

FIGURE 5 | Growth responses of Arabidopsis Col0 and SPLCV-JS C4 transgenic lines to $1 \mu$ M epibrassinolide (BL). (A,B) Real-time qRT-PCR analysis of the expression level of brassinosteroid biosynthetic genes CPD and DWF4 in ColO and SPLCV-JS C4 transgenic lines with or without BL treatment. (C) Phenotypic status of Col0 and C4-11, C4-36, and C4-37 plant lines on agar medium 5 days after the BL treatment. (D) Root growth repression of Col0 and C4-11, C4-36, and C4-37 plant lines by the BL treatment. Data presented as mean $\pm \mathrm{SD}$. Asterisks indicate a statistically significant difference compared with the wild-type value according to the Student's $t$-test $(P<0.05)$.

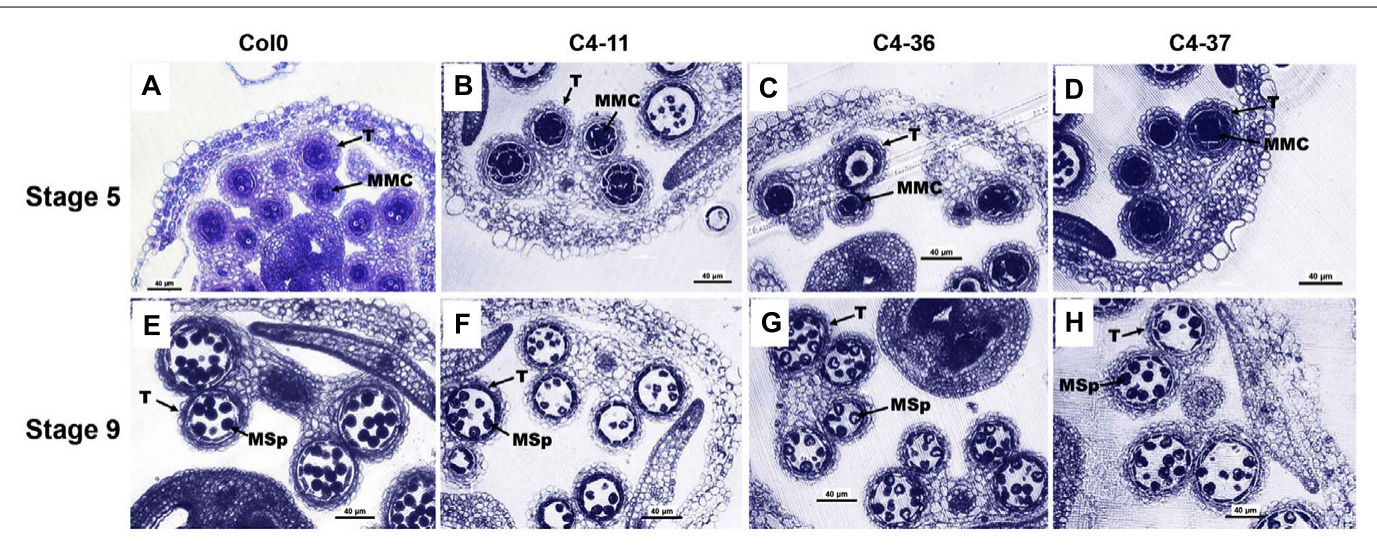

FIGURE 6 | Microspores and anther development in the wild-type Col0 and SPLCV-JS C4 transgenic plants. (A-D) Anthers at stage 5 . (E-H) Anthers at stage 9. Col0 (A,E); C4-11 (B,F); C4-36 (C,G); C4-37 (D,H). Scale bar = 40 m. MMC, microspore mother cell; Msp, microspore; and T, tapetum.

the abnormal development of tapetum may be an important reason for the reduced male fertility in the $\mathrm{C} 4$ transgenic plants.

The expression levels of key genes related to the genetic program controlling anther and pollen development in Arabidopsis (Ma, 2005) were analyzed in the buds containing anthers at approximately stages 7-9 of the C4 transgenic lines using qRT-PCR to further identify the effect of $\mathrm{C} 4$ on male fertility. The expression levels of SPROROCYTELESS/NOZZLE (SPL/NZZ), DYSFUNCTIONAL TAPETUM 1 (DYT1), DEFECTIVE IN TAPEL DEVELOPMENT AND FUNCTION 1 (TDF1), ABORTED MICROSPORES (AMS), MYB domain protein 103 (MYB103), MALE STERILITY 1 (MS1), and MALE STERILITY 2 (MS2) (Ye et al., 2010) were significantly lower than 

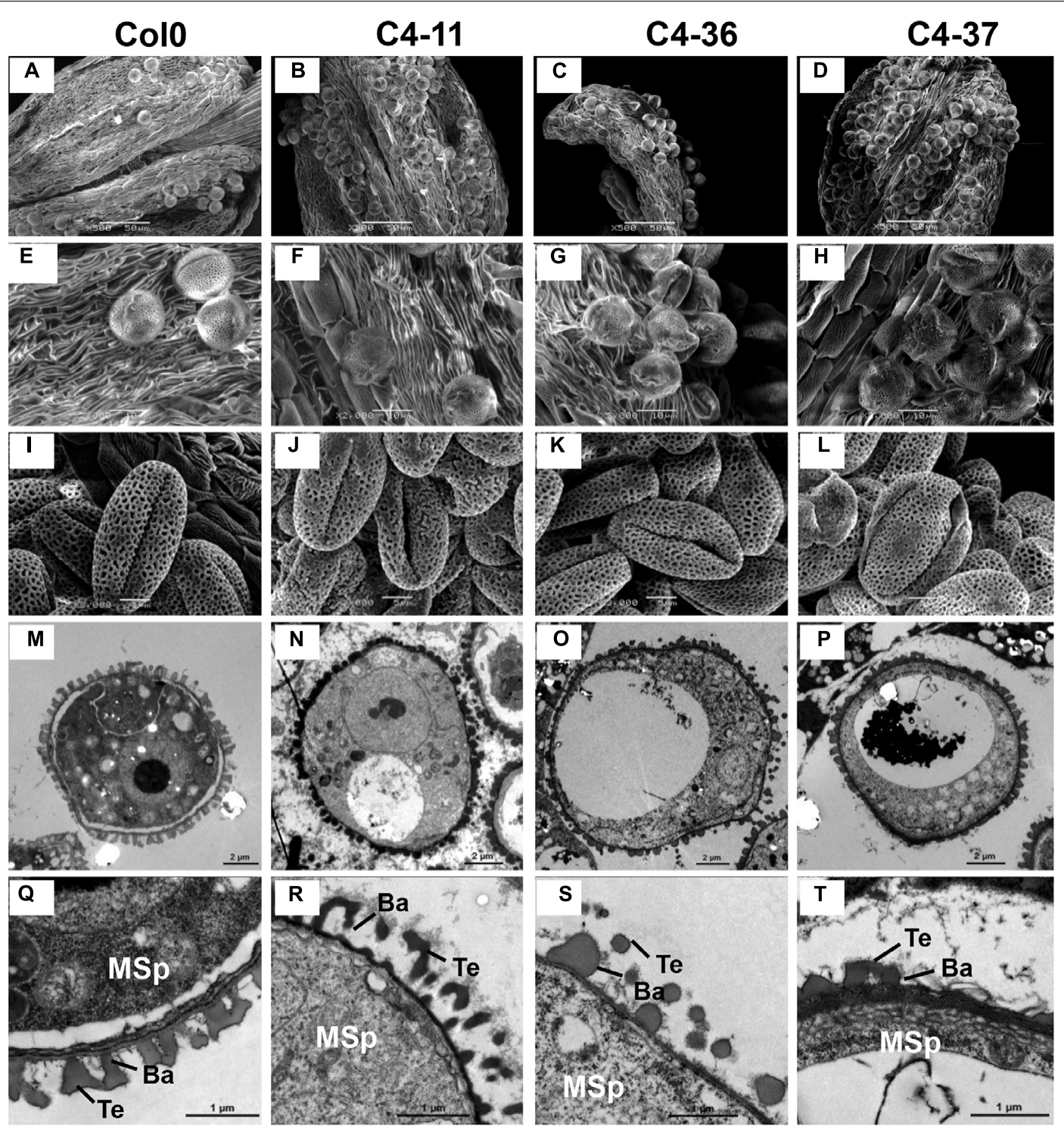

FIGURE 7| TEM observations of microspores at stage 9 and SEM examination of dehiscent anthers and pollen grains from SPLCV-JS C4 transgenic lines and the wild type. (A-H) Scanning electron micrographs of dehiscent anthers of Col0, C4-11, C4-36, and C4-37. (I-L) Scanning electron micrographs of mature pollen grains. (M-T) Transmission electron micrographs of transverse section of microspores and the exine ultrastructure of pollen wall at stage 9 . Scale bar $=50 \mu \mathrm{m}$ (A-D), $10 \mu \mathrm{m}$ (E-H), $5 \mu \mathrm{m}$ (I-L), $2 \mu \mathrm{m}$ (M-P), $1 \mu \mathrm{m}$ (Q-T). Ba, Bacula; Msp, microspore; and Te, tectum.

that of the wild type (Figure 9). Furthermore, the expressions of several MS1 downstream genes were measured (Ye et al., 2010). It was found that the expression levels of At4g28395, At3g42960, At3g51590, At1g07340, At3g23770, At1g61110, and At5g62320 in C4-11, C4-36, and C4-37 were lower than that in the wild type, while the expression level of At2g18550 was higher than the wild type (Figure 9). This was in accordance with the case of the BR mutant $c p d$ reported previously (Ye et al., 2010). Since AtBES1 could directly bind to the promoter regions of genes $S P L / N Z Z$, TDF1, AMS, MS1, and MS2 to regulate their expression (Ye et al., 2010), C4-activated AtBES1/AtBZR1 modified the expression pattern of downstream genes that were essential for anther and pollen development, consequently affecting the development of tapetum and microspore and leading to reduced male fertility and decreased seed number in the transgenic lines.

\section{DISCUSSION}

Geminivirus C4 proteins are small proteins with diverse functions, and play an important role in virus-plant interaction (Hanley-Bowdoin et al., 2013). The C4 proteins of several geminiviruses have been reported to be symptom-determinant and counteract posttranscriptional gene silencing (Stanley and Latham, 1992; Rigden et al., 1994; Fondong et al., 2007). They are also involved in virus migration (Jupin et al., 1994; Rojas 


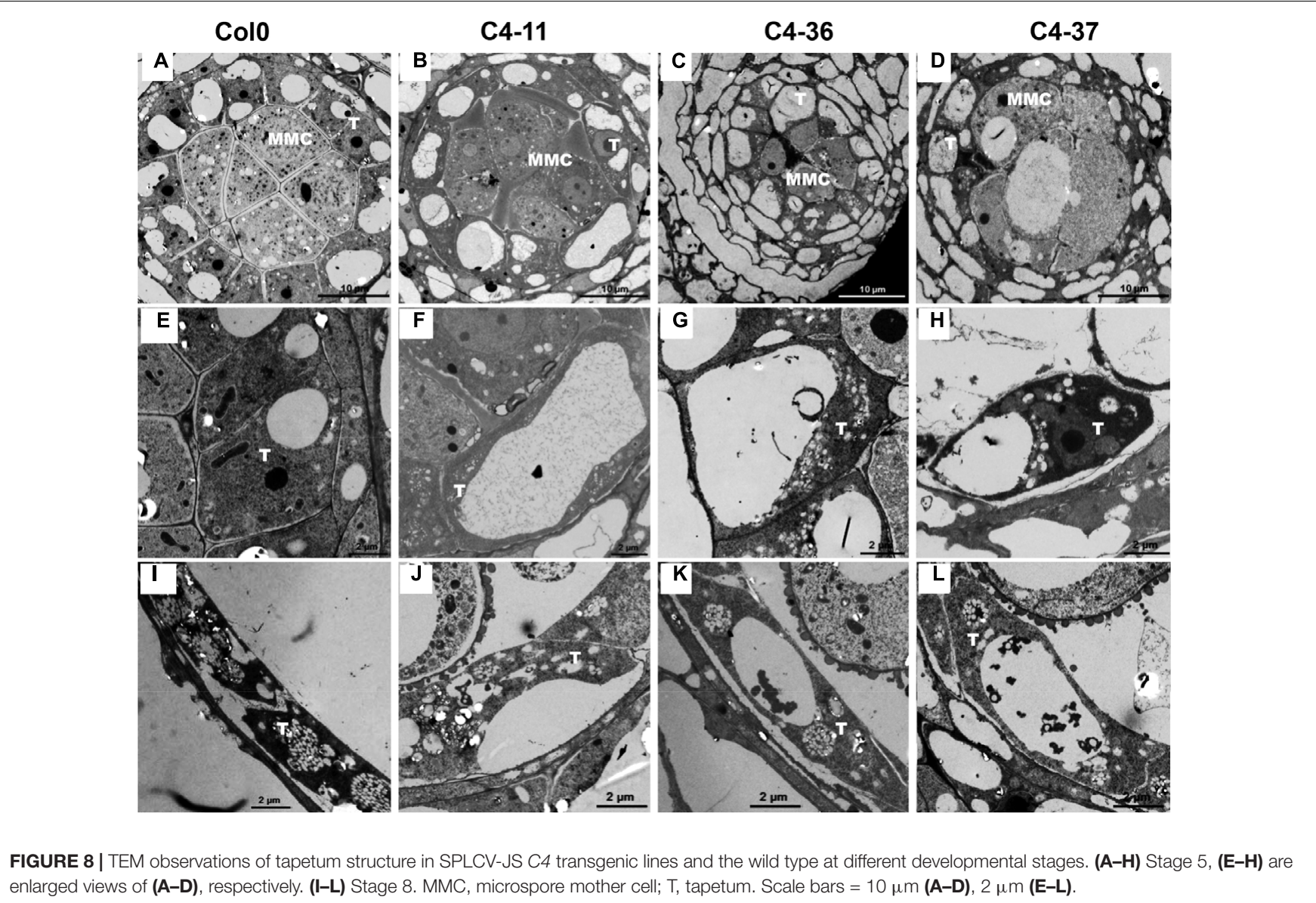

enlarged views of (A-D), respectively. (I-L) Stage 8. MMC, microspore mother cell; T, tapetum. Scale bars = $10 \mu \mathrm{m}$ (A-D), $2 \mu \mathrm{m}$ (E-L)

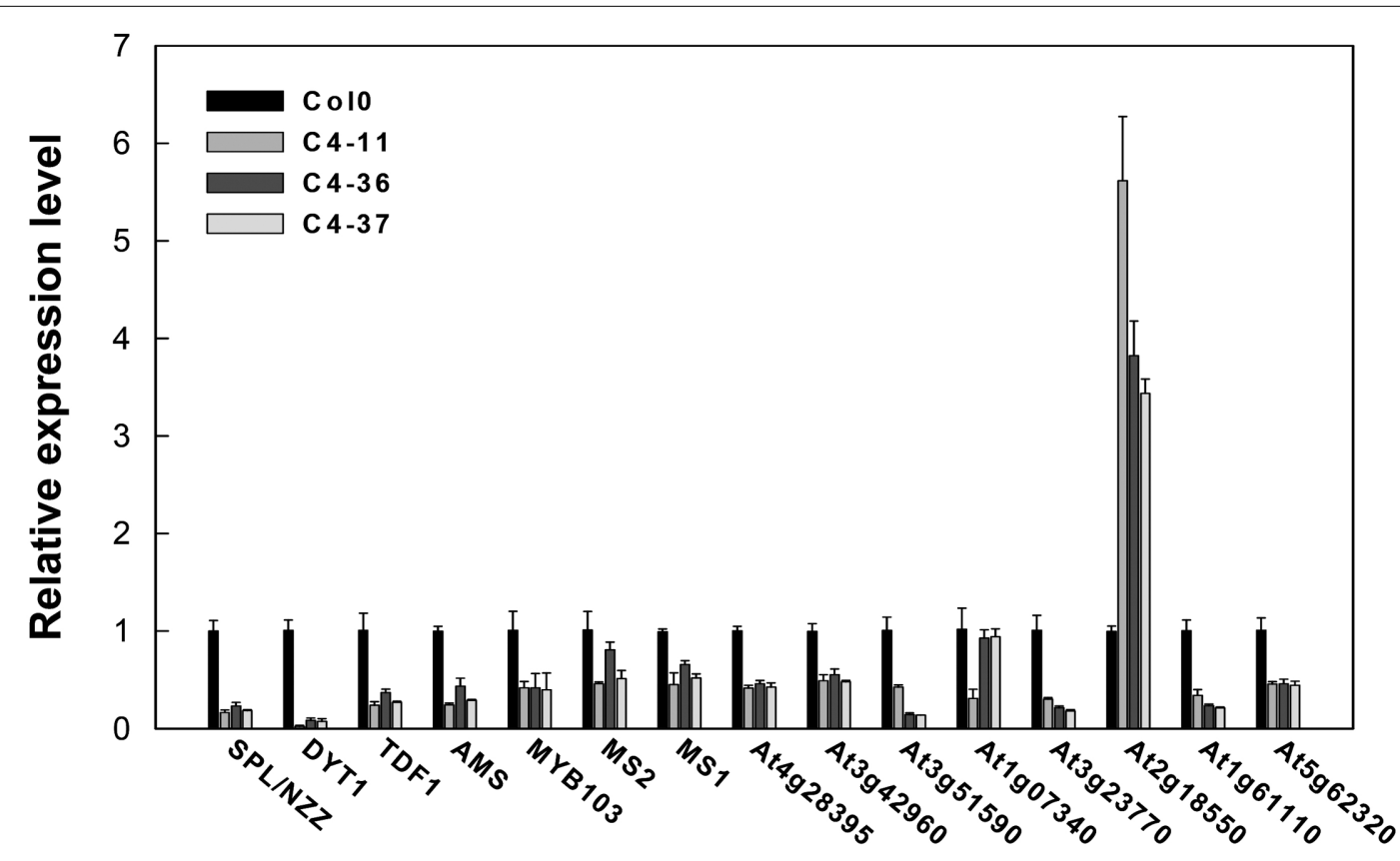

FIGURE 9 | Real-time qRT-PCR analyses of the expression level of several essential genes in anther and pollen development in SPLCV C4 transgenic lines and the wild-type ColO. The expression level of each gene referred to Col0. The U-BOX gene At5g15400 was used as an internal reference. Data presented as mean \pm SD. 
et al., 2001; Teng et al., 2010). Recent studies also found that the C4 protein of BCTV and ToLCV interacts with the plant BRsignaling pathway (Piroux et al., 2007; Dogra et al., 2009; MillsLujan and Deom, 2010; Mills-Lujan et al., 2015). The present study demonstrated that the ectopic expression of SPLCV-JS C4 protein in Arabidopsis caused severe phenotypic changes and reduced male fertility. The BiFC assay showed that the $\mathrm{C} 4$ could interact with AtBIN2 mainly in the plasma membrane to activate AtBES1/AtBZR1 components, so that they could enter the nucleus and activate transcription of BR-response genes.

All transgenic Arabidopsis plant lines that showed severe diseased-like phenotypes were the multi-copy insertion of transgene (Supplementary Figure S2A), indicating that singlecopy insertion of SPLCV-JS C4 in Arabidopsis might be lethal. Transgenic Arabidopsis was reported to be produced only when the expression of the BCTV C4 gene was driven by an inducible promoter (Mills-Lujan and Deom, 2010). Transgenic sweet potato plants could not be produced using constitutive expression of SPLCV-JS C4, indicating that the C4 protein might be toxic to the embryogenic callus or during somatic embryogenesis.

Geminivirus C4 interacts with SHAGGY-like kinases (Piroux et al., 2007; Dogra et al., 2009; Mills-Lujan et al., 2015). The interaction of SPLCV-JS C4 protein and AtBIN2 in this study enabled the release of AtBES1/AtBZR1 in the nucleus to upregulate the BR-signaling pathway. AtBIN2 has been reported to phosphorylate BCTV C4 at threonine and serine residues (Piroux et al., 2007), specifically serine 49 (Mills-Lujan et al., 2015), which was present in the SPLCV-JS C4. By analogy, SPLCV-JS C4 might act as a competitive substrate of AtBIN2 to suppress the phosphorylation of AtBES1/AtBZR1, and the unphosphorylated AtBES1/AtBZR1 was released and accumulated in the nucleus to regulate the downstream genes expression (Figure 10), which was verified by BR-responsive target genes such as $C P D$ and DWF4 (Figures 5A,B). Previous studies have reported that the $\mathrm{S}^{49}$ residue of BCTV C4 was critical for its interaction with AtSKs and phenotype induction (Mills-Lujan et al., 2015). Besides $S^{49}$, Piroux et al. (2007) also identified additional amino acids $\mathrm{P}^{44}$, $\mathrm{A}^{45}$, and $\mathrm{P}^{50}$ within the central domain of BCTV contributing to the phenotype. In the SPLCV-JS C4 truncations, the C4N55 containing $\mathrm{P}^{44}, \mathrm{~S}^{49}$, and $\mathrm{P}^{50}$ did not induce obvious altered phenotypes as those induced by intact C4 (Supplementary Figure S3), and C4N55 could not interact with AtBIN2 in the yeast two-hybrid assay (Figure 3A). Instead, the C4C31 could induce slight phenotype changes in the transgenic Arabidopsis plants (Supplementary Figure S3) and interact with AtBIN2 weakly in the yeast two-hybrid assay (Figure 3A). Further experiments are needed to reveal whether and which sites of SPLCV-JS C4 protein are phosphorylated by AtBIN2. Besides AtBIN2, SPLCV-JS C4 may also interact with other AtSKs as the case of BCTV C4 protein (Deom and Mills-Lujan, 2015; Mills-Lujan et al., 2015).

Brassinosteroids are essential regulators of plant growth and development. Many studies have shown that mutations in BR synthesis and signaling components are deleterious for plant growth and reproduction. Ye et al. (2010) demonstrated reduced pollen number, viability, and efficiency in the male reproductive systems of a series of BR biosynthetic and signaling mutants. The AtBES1 targeted genes: SPL/NZZ, TDF1, AMS, MS1, and MS2 that are essential for anther and microspore development (Ye et al., 2010) indeed were found to be down-regulated in their expression in the SPLCV-JS C4 transgenic Arabidopsis (Figure 9). Histological, SEM, and TEM analyses of microspores and pollens in SPLCV-JS C4 transgenic Arabidopsis plants also revealed that the expression of $\mathrm{C} 4$ caused abnormal tapetum development and pollen maturation, leading to defective exine pattern formation of microspores and pollen release (Figures 7, 8), a scenario similar to the BR-related mutants (Ye et al., 2010). Therefore, reduced male fertility in SPLCV-JS C4 transgenic Arabidopsis plants is likely a result of C4-mediated activation of BR-signaling pathway. Interestingly, the At 2 g18550, which encodes homeobox-leucine zipper protein ATHB-21 that interacts with ABA-responsive element binding factor AREB2/ABF4 (Comelli et al., 2012), was significantly upregulated, indicating the crosstalk between BR and ABA signaling pathways (Zhang et al., 2009).

The retention of AtBZR1 in the nucleus as a consequence of SPLCV-JS C4-AtBIN2 interaction also caused transcriptional regulation of many $B R$-regulated $B Z R 1$ target (BRBT) genes. These BRBT genes are related to a wide range of cellular activities and biological processes, including responses to other hormones and environmental stress (Sun et al., 2010), for example, the auxin response factor genes (McSteen and Zhao, 2008); genes encoding
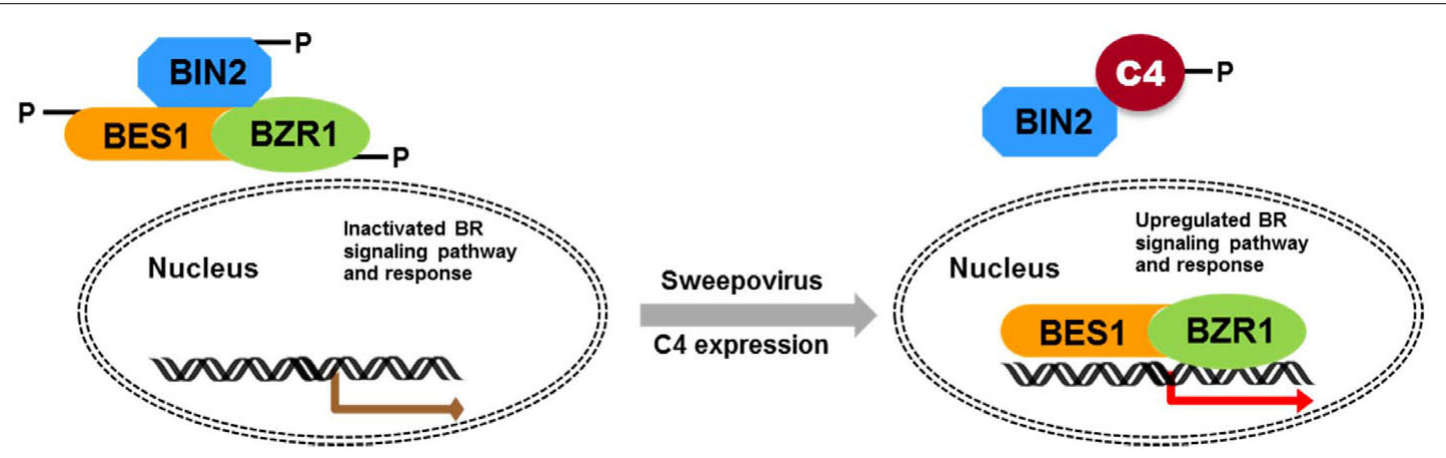

FIGURE 10 | Schematic illustration of SPLCV-JS C4 function in activating BR-signaling pathway. In Arabidopsis expressing the sweepovirus C4, AtBIN2 phosphorylated SPLCV-JS C4 to suppress the phosphorylation of AtBES1/AtBZR1, and the unphosphorylated AtBES1/AtBZR1 was released and accumulated in the nucleus to regulate downstream genes expression. 
auxin/indole-3-acetic acid (IAAs) protein (Nakamura et al., 2003) involved in auxin signaling pathway; abiotic-responsive genes CBF2, RD29a; and biotic-responsive gene WRKY17 (Sun et al., 2010). The expression of ARF6, CBF2, RD29a, and WRKY17 in $C 4$ transgenic lines was repressed compared with the wild type, while the expression of IAA19 increased (Supplementary Figure S5), similar to the BL-induced situations (Sun et al., 2010). These changes may worsen the plant phenotype in the C4-expressing lines. The present data indicate that the SPLCV-JS C4 interfered with the BR-signaling pathway, paving a way of understanding how sweepoviruses interacted with their host plants.

Brassinosteroid has already been found to induce disease resistance in plants (Nakashita et al., 2003). The interaction between geminivirus $\mathrm{C} 4$ protein and AtBIN2 might be a host defense reaction to tag the $\mathrm{C} 4$ protein for degradation (Piroux et al., 2007). Mills-Lujan et al. (2015) reported that plasma membrane-associated BCTV C4 protein interacted with and co-opted multiple AtSKs to promote its own phosphorylation and activation to subsequently compromise cell cycle control. Therefore, SPLCV-JS C4 protein can facilitate its virus infection through interacting with the BR-signaling pathway, but the interaction can also activate the steroid hormonemediated disease resistance of host plants. Nevertheless, whether SPLCV-JS C4 interacts with other AtSKs or the BRsignaling pathway of sweet potato can be activated by SPLCV-JS during natural infection remains to be investigated in future studies.

Outbreaks of sweepovirus disease have been reported worldwide and caused substantial yield losses and cultivar decline in sweet potato. Nevertheless, sweepoviruses have not drawn much attention of virologists. Only recently several strains or isolates were identified and their infection assays established (Trenado et al., 2011; Bi and Zhang, 2012, 2014).

\section{REFERENCES}

Albuquerque, L. C., Inoue-Nagata, A. K., Pinheiro, B., Resende, R. O., Moriones, E., and Navas-Castillo, J. (2012). Genetic diversity and recombination analysis of sweepoviruses from Brazil. Virol. J. 9, 241. doi: 10.1186/1743-422X-9-241

Bi, H., and Zhang, P. (2012). Molecular characterization of two sweepoviruses identified from China and infectivity evaluation of cloned SPLCV-JS in Nicotiana benthamiana. Arch. Virol. 157, 441-454. doi: 10.1007/s00705-0111194-6

Bi, H., and Zhang, P. (2014). Agroinfection of sweet potato by vacuum infiltration of an infectious sweepovirus. Virol. Sin. 29, 148-154. doi: 10.1007/s12250-0143430- 1

Clark, C. A., Davis, J. A., Abad, J. A., Cuellar, W. J., Fuentes, S., Kreuze, J. F., et al. (2012). Sweetpotato viruses: 15 years of progress on understanding and managing complex diseases. Plant Dis. 96, 168-185. doi: 10.1094/PDIS-07-110550

Clark, C. A., and Hoy, M. W. (2006). Effects of common viruses on yield and quality of Beauregard sweet potato in Louisiana. Plant Dis. 90, 83-88. doi: 10.1094/PD-90-0083

Comelli, R. N., Welchen, E., Kim, H. J., Hong, J. C., and Gonzalez, D. H. (2012). Delta subclass HD-Zip proteins and a B-3 AP2/ERF transcription factor interact with promoter elements required for expression of the Arabidopsis cytochrome c oxidase 5b-1 gene. Plant Mol. Biol. 80, 157-167. doi: 10.1007/s11103-0129935-9

Cuellar, W. J., Galvez, M., Fuentes, S., Tugume, J., and Kreuze, J. (2014). Synergistic interactions of begomoviruses with sweet potato chlorotic stunt virus (genus
An increase in the understanding of the virus-plant interaction is essential for developing durable and sustainable strategies for biotechnological control of virus pandemics. Therefore, the present study not only disclosed how the SPLCV-JS C4 protein interacted with plant hormone signaling pathway and subsequently affected plant growth and reproduction, but also confirmed that the $\mathrm{C} 4$ protein could be used as a target gene for engineering crops resistant to sweepoviruses.

\section{AUTHOR CONTRIBUTIONS}

HB performed most of the experiments and drafted the manuscript. WF conducted part of yeast two-hybrid assay. PZ coordinated and designed the study and revised the article.

\section{FUNDING}

This work was supported by the National Natural Science Foundation of China (31501356), Collaborative Innovation Action - Agricultural Science and Technology Innovation Program of Chinese Academy of Agricultural Sciences (CAASXTCX2016009), XDPB04 of CAS, and the National Key Technology Research and Development Program of China (2015BAD15B01).

\section{SUPPLEMENTARY MATERIAL}

The Supplementary Material for this article can be found online at: http://journal.frontiersin.org/article/10.3389/fpls.2017.01689/ full\#supplementary-material

Crinivirus) in sweetpotato (Ipomoea batatas L.). Mol. Plant Pathol. 16, 459-471. doi: $10.1111 / \mathrm{mpp} .12200$

Deom, C. M., and Mills-Lujan, K. (2015). Toward understanding the molecular mechanism of a geminivirus C4 protein. Plant Signal. Behav. 10:e1109758. doi: 10.1080/15592324.2015.1109758

Doble, B. W., and Woodgett, J. R. (2003). GSK-3: tricks of the trade for a multi-tasking kinase. J. Cell Sci. 116, 1175-1186. doi: 10.1242/jcs.00384

Dogra, S., Eini, O., Rezaian, M. A., and Randles, J. W. (2009). A novel shaggy-like kinase interacts with the tomato leaf curl virus pathogenicity determinant C4 protein. Plant Mol. Biol. 71, 25-38. doi: 10.1007/s11103-009-9506-x

Fàbregas, N., and Caño-Delgado, A. I. (2014). Turning on the microscope turret: a new view for the study of brassinosteroid signaling in plant development. Physiol. Plant. 151, 172-183. doi: 10.1111/ppl.12130

Fondong, V. N., Reddy, R. C., Lu, C., Hankoua, B., Felton, C., Czymmek, K., et al. (2007). The consensus $N$-myristoylation motif of a geminivirus AC4 protein is required for membrane binding and pathogenicity. Mol. Plant Microbe Interact. 20, 380-391. doi: 10.1094/MPMI-20-4-0380

Gampala, S. S., Kim, T. W., He, J., Tang, W., Deng, Z., Bai, M., et al. (2007). An essential role for 14-3-3 proteins in brassinosteroid signal transduction in Arabidopsis. Dev. Cell 13, 177-189. doi: 10.1016/j.devcel.2007.06.009

Gibson, R. W., and Kreuze, J. F. (2015). Degeneration in sweetpotato due to viruses, virus-cleaned planting material and reversion: a review. Plant Pathol. 64, 1-15. doi: $10.1111 /$ ppa.12273

Gopal, P., Pravin Kumar, P., Sinilal, B., Jose, J., Kasin Yadunandam, A., and Usha, R. (2007). Differential roles of $C 4$ and $\beta C 1$ in mediating suppression of posttranscriptional gene silencing: evidence for transactivation by the $\mathrm{C} 2$ of Bhendi 
yellow vein mosaic virus, a monopartite begomovirus. Virus Res. 123, 9-18. doi: 10.1016/j.virusres.2006.07.014

Gutierrez, C. (2002). Strategies for geminivirus DNA replication and cell cycle interference. Physiol. Mol. Plant Pathol. 60, 219-230. doi: 10.1006/pmpp.2002. 0401

Hanley-Bowdoin, L., Bejarano, E. R., Robertson, D., and Mansoor, S. (2013). Geminiviruses: masters at redirecting and reprogramming plant processes. Nat. Rev. Microbiol. 11, 777-788. doi: 10.1038/nrmicro3117

He, J. X., Gendron, J. M., Sun, Y., Gampala, S. S., Gendron, N., Sun, C. Q., et al. (2005). BZR1 is a transcriptional repressor with dual roles in brassinosteroid homeostasis and growth responses. Science 307, 1634-1638. doi: 10.1126/ science. 1107580

Jupin, I., De Kouchkovsky, F., Jouanneau, F., and Gronenborn, B. (1994). Movement of tomato yellow leaf curl geminivirus (TYLCV): involvement of the protein encoded by ORF C4. Virology 204, 82-90. doi: 10.1006/viro.1994.1512

Krake, L. R., Rezaian, M. A., and Dry, I. B. (1998). Expression of the tomato leaf curl geminivirus $\mathrm{C} 4$ gene produces viruslike symptoms in transgenic plants. Mol. Plant Microbe Interact. 11, 413-417. doi: 10.1094/MPMI.1998.11.5.413

Lai, J., Chen, H., Teng, K., Zhao, Q., Zhang, Z., Li, Y., et al. (2009). RKP, a RING finger E3 ligase induced by BSCTV C4 protein, affects geminivirus infection by regulation of the plant cell cycle. Plant J. 57, 905-917. doi: 10.1111/j.1365-313X. 2008.03737.x

Li, H., Lin, Y., Heath, R. M., Zhu, M. X., and Yang, Z. (1999). Control of pollen tube tip growth by a Rop GTPase-dependent pathway that leads to tip-localized calcium influx. Plant Cell 11, 1731-1742. doi: 10.1105/tpc.11.9.1731

Li, J., Nam, K. H., Vafeados, D., and Chory, J. (2001). BIN2, a new brassinosteroidinsensitive locus in Arabidopsis. Plant Physiol. 127, 14-22. doi: 10.1104/pp. 127.1.14

Li, X. (2011). Pollen fertility/viability assay using FDA staining. Bioprotocol Bio101:e75. doi: 10.21769/BioProtoc.75

Ling, K. S., Jackson, D. M., Harrison, H., Simmons, A. M., and PesicVanEsbroeck, Z. (2010). Field evaluation on yield effects of USA heirloom sweet potato cultivars infected by sweet potato leaf curl virus. Crop Prot. 29, 757-765. doi: 10.1016/j.cropro.2010.02.017

Liu, Q., Zhang, Z., Li, J., Qiao, Q., Qin, Y., Zhang, D., et al. (2014). Complete genome sequence of a novel monopartite begomovirus infecting sweet potato in China. Arch. Virol. 159, 1537-1540. doi: 10.1007/s00705-013-1958-2

Livak, K. J., and Schmittgen, T. D. (2001). Analysis of relative gene expression data using real-time quantitative PCR and the $2^{-\Delta \Delta C_{\mathrm{T}}}$ method. Methods 25 , 402-408. doi: 10.1006/meth.2001.1262

Lozano-Durán, R., Rosas-Díaz, T., Gusmaroli, G., Luna, A. P., Taconnat, L., Deng, X. W., et al. (2011). Geminiviruses subvert ubiquitination by altering CSNmediated derubylation of SCF E3 ligase complexes and inhibit jasmonate signaling in Arabidopsis thaliana. Plant Cell 23, 1014-1032. doi: 10.1105/tpc. 110.080267

Lucioli, A., Perla, C., Berardi, A., Gatti, F., Spanò, L., and Tavazza, M. (2016). Transcriptomics of tomato plants infected with TYLCSV or expressing the central TYLCSV Rep protein domain uncover changes impacting pathogen response and senescence. Plant Physiol. Biochem. 103, 61-70. doi: 10.1016/j. plaphy.2016.02.034

Ma, H. (2005). Molecular genetic analyses of microsporogenesis and microgametogenesis in flowering plants. Annu. Rev. Plant Biol. 56, 393-434. doi: 10.1146/annurev.arplant.55.031903.141717

McSteen, P., and Zhao, Y. (2008). Plant hormones and signaling: common themes and new developments. Dev. Cell 14, 467-473. doi: 10.1016/j.devcel.2008. 03.013

Mills-Lujan, K., Andrews, D. L., Chou, C. W., and Deom, C. M. (2015). The roles of phosphorylation and SHAGGY-Like protein kinases in geminivirus C4 protein induced hyperplasia. PLOS ONE 10:e0122356. doi: 10.1371/journal. pone. 0122356

Mills-Lujan, K., and Deom, C. M. (2010). Geminivirus C4 protein alters Arabidopsis development. Protoplasma 239, 95-110. doi: 10.1007/s00709-0090086-z

Nakamura, A., Higuchi, K., Goda, H., Fujiwara, M. T., Sawa, S., Koshiba, T., et al. (2003). Brassinolide induces IAA5, IAA19, and DR5, a synthetic auxin response element in Arabidopsis, implying a cross talk point of brassinosteroid and auxin signaling. Plant Physiol. 133, 1843-1853. doi: 10.1104/pp.103.030031
Nakashita, H., Yasuda, M., Nitta, T., Asami, T., Fujioka, S., Arai, Y., et al. (2003). Brassinosteroid functions in a broad range of disease resistance in tobacco and rice. Plant J. 33, 887-898. doi: 10.1046/j.1365-313X.2003.01675.x

Pardina, P. R., Luque, A., Nome, C., Colomba, E. L., Delgado, S. F., and Di Feo, L. (2012). First report of Sweet potato leaf curl virus infecting sweet potato in Argentina. Australas. Plant Dis. Notes 7, 157-160. doi: 10.1007/s13314-0120073-7

Piroux, N., Saunders, K., Page, A., and Stanley, J. (2007). Geminivirus pathogenicity protein C4 interacts with Arabidopsis thaliana shaggy-related protein kinase AtSK $\eta$, a component of the brassinosteroid signalling pathway. Virology 362, 428-440. doi: 10.1016/j.virol.2006.12.034

Rigden, J. E., Krake, L. R., Rezaian, M. A., and Dry, I. B. (1994). ORF C4 of tomato leaf curl geminivirus is a determinant of symptom severity. Virology 204, 847-850. doi: 10.1006/viro.1994.1606

Rojas, M. R., Jiang, H., Salati, R., Xoconostle-Cazares, B., Sudarshana, M. R., Lucas, W. J., et al. (2001). Functional analysis of proteins involved in movement of the monopartite begomovirus, Tomato yellow leaf curl virus. Virology 291, 110-125. doi: 10.1006/viro.2001.1194

Ryu, H., Kim, K., Cho, H., Park, J., Choe, S., and Hwang, I. (2007). Nucleocytoplasmic shuttling of BZR1 mediated by phosphorylation is essential in Arabidopsis brassinosteroid signaling. Plant Cell 19, 2749-2762. doi: 10.1105/ tpc.107.053728

Sambrook, J., Fritsch, E. F., and Maniatis, T. (2001). Molecular Cloning: A Laboratory Manual, 3rd Edn. Cold Spring Harbor: Cold Spring Harbor Laboratory Press.

Sanders, P. M., Bui, A. Q., and Goldberg, R. B. (1999). Anther developmental defects in Arabidopsis thaliana male-sterile mutants. Sex. Plant Reprod. 11, 297-322. doi: 10.1007/s004970050158

Simmons, A. M., Ling, K. S., Harrison, H. F., and Jackson, D. M. (2009). Sweet potato leaf curl virus: efficiency of acquisition, retention and transmission by Bemisia tabaci (Hemiptera: Aleyrodidae). Crop Prot. 28, 1007-1011. doi: 10.1016/j.cropro.2009.06.011

Soni, R., and Murray, J. A. H. (1994). Isolation of intact DNA and RNA from plant tissues. Anal. Biochem. 218, 474-476. doi: 10.1006/abio.1994.1214

Stanley, J., and Latham, J. R. (1992). A symptom variant of beet curly top geminivirus produced by mutation of open reading frame C4. Virology 190, 506-509. doi: 10.1016/0042-6822(92)91243-N

Sun, Y., Fan, X., Cao, D., Tang, W., He, K., Zhu, J., et al. (2010). Integration of brassinosteroid signal transduction with the transcription network for plant growth regulation in Arabidopsis. Dev. Cell 19, 765-777. doi: 10.1016/j.devcel. 2010.10.010

Tamura, K., Dudley, J., Nei, M., and Kumar, S. (2007). MEGA4: molecular Evolutionary Genetics Analysis (MEGA) software version 4.0. Mol. Biol. Evol. 24, 1596-1599. doi: 10.1093/molbev/msm092

Teng, K., Chen, H., Lai, J., Zhang, Z., Fang, Y., Xia, R., et al. (2010). Involvement of $\mathrm{C} 4$ protein of beet severe curly top virus (family Geminiviridae) in virus movement. PLOS ONE 5:e11280. doi: 10.1371/journal.pone.0011280

Trenado, H. P., Orílio, A. F., Márquez-Martín, B., Moriones, E., and NavasCastillo, J. (2011). Sweepoviruses cause disease in sweet potato and related Ipomoea spp.: fulfilling koch's postulates for a divergent group in the genus Begomovirus. PLOS ONE 6:e27329. doi: 10.1371/journal.pone.0027329

Vanitharani, R., Chellappan, P., Pita, J. S., and Fauquet, C. M. (2004). Differential roles of AC2 and AC4 of cassava geminiviruses in mediating synergism and suppression of posttranscriptional gene silencing. J. Virol. 78, 9487-9498. doi: 10.1128/JVI.78.17.9487-9498.2004

Vert, G., and Chory, J. (2006). Downstream nuclear events in brassinosteroid signalling. Nature 441, 96-100. doi: 10.1038/nature04681

Wang, Z. Y., Bai, M. Y., Oh, E., and Zhu, J. Y. (2012). Brassinosteroid signaling network and regulation of photomorphogenesis. Annu. Rev. Genet. 46, 701-724. doi: 10.1146/annurev-genet-102209-163450

Wasswa, P., Otto, B., Maruthi, M. N., Mukasa, S. B., Monger, W., and Gibson, R. W. (2011). First identification of a sweet potato begomovirus (sweepovirus) in Uganda: characterization, detection and distribution. Plant Pathol. 60, 1030-1039. doi: 10.1111/j.1365-3059.2011.02464.x

Yang, C., Zhang, C., Lu, Y., Jin, J., and Wang, X. (2011). The mechanisms of brassinosteroids' action: from signal transduction to plant development. Mol. Plant 4, 588-600. doi: 10.1093/mp/ssr020 
Ye, Q., Zhu, W., Zhang, S., Yin, Y., Ma, H., and Wang, X. (2010). Brassinosteroids control male fertility by regulating the expression of key genes involved in Arabidopsis anther and pollen development. Proc. Natl. Acad. Sci. U.S.A. 107, 6100-6105. doi: 10.1073/pnas.09123 33107

Youn, J. H., and Kim, T. W. (2015). Functional insights of plant GSK3-like kinases: multi-taskers in diverse cellular signal transduction pathways. Mol. Plant. 8, 552-565. doi: 10.1016/j.molp.2014.12.006

Zhang, S., Cai, Z., and Wang, X. (2009). The primary signaling outputs of brassinosteroids are regulated by abscisic acid signaling. Proc. Natl. Acad. Sci. U.S.A. 106, 4543-4548. doi: 10.1073/pnas. 0900349106

Zhang, X., Henriques, R., Lin, S., Niu, Q., and Chua, N. (2006). Agrobacterium-mediated transformation of Arabidopsis thaliana using the floral dip method. Nat. Protoc. 1, 641-646. doi: 10.1038/nprot. 2006.97

Conflict of Interest Statement: The authors declare that the research was conducted in the absence of any commercial or financial relationships that could be construed as a potential conflict of interest.

Copyright (C) 2017 Bi, Fan and Zhang. This is an open-access article distributed under the terms of the Creative Commons Attribution License (CC BY). The use, distribution or reproduction in other forums is permitted, provided the original author(s) or licensor are credited and that the original publication in this journal is cited, in accordance with accepted academic practice. No use, distribution or reproduction is permitted which does not comply with these terms. 